\title{
Using Bacterial Foraging Algorithm to Design Optimal Power System Stabilizer and Comparisons with Genetic Algorithm and Particle Swarm Optimization
}

\author{
Muwaffaq I. Alomoush \\ Department of Electrical Power Engineering, Hijjawi Faculty for Engineering Technology, Yarmouk University \\ Irbid, Jordan \\ Email: ma@yu.edu.jo
}

\begin{abstract}
The Bacterial Foraging (BF) is a global optimization algorithm that imitates the foraging behavior of Escherichia coli (E. coli) bacteria. Foraging habit of individuals and groups of E. coli bacteria for nutrients is modeled as a distributed optimization process. This paper applies this powerful algorithm to design an optimal controller for a single-machine-infinite-bus (SMIB) system equipped with a power system stabilizer (PSS). The main target of the optimal design is to improve the dynamic and steady-state responses when the system is subjected to sever typical disturbances. The BF-based design of the PSS will be also compared with the designs obtained by the two commonly used global optimizers; the Genetic Algorithm (GA) and Particle Swarm Optimization (PSO). Simulation results presented in the paper indicate robustness and superiority of the proposed $\mathrm{BF}$ algorithm to solve the design problem, where the optimal BF-based controller can significantly stabilize the system and efficiently dampen oscillations under disturbances. Results also disclose that $\mathrm{BF}$ has the capability to offer a much better-quality global solution with better convergence characteristics than GA and PSO. Results show that the BF-based controller has better impact on system performance in terms of damping the electromechanical oscillations of the rotor angle, rotational speed, electromagnetic torque, and terminal voltage under various disturbance conditions and its damping speed is considerably faster. The significant improvement associated with the BFbased PSS is due to the fact that the BF algorithm leads to a better global solution of the optimization problem under study.
\end{abstract}

Keywords: Bacterial foraging, optimal controllers, power system control, small-signal stability SMIB

\section{$1 \quad$ Introduction}

An interconnected power system should be capable to generate, transmit and distribute electric power to end-users while keeping an economic and a reliable operation. The system should simultaneously preserve quality of power, voltage magnitude, frequency level, and tie-line power flows within the acceptable limits [1].

When an interconnected power system is subjected to possible disturbances such as sudden load changes, parameter variations, switching operations, faults, system variables undergo oscillations. Some electromechanical oscillations of small magnitude and low frequency exist in the interconnected power system and often persist for long periods of time and might limit power transfer capability of the system [2]. A power system that has a low or a negative damping is subjected to spontaneous appearance of large power oscillations.

Interconnected power systems are subjected to two distinct types of system oscillations, which are local mode oscillations and inter-area mode oscillations [2, 3]. In the former type, oscillations are localized at one power plant, where units at a generating station swing with respect to the rest of the power system. The later type is associated with a swinging of many machines in an area of the system against machines in other areas [3].

Several devices and methods are available to increase damping in a power system such as PSS. PSS is 
an auxiliary controller added to a synchronous generator to be used in conjunction with machine excitation system to generate supplementary feedback stabilizing control signal that suppresses all types of oscillations when operating conditions of a power system change due to disturbances. Therefore, PSS enhances system damping and improves power transfer limits in order to maintain a reliable operation of a power system [1-5]. Nowadays, majority of generators are equipped with rapid responding, high-gain voltage regulator and excitation systems to automatically control the terminal voltage when the system is subjected to perturbations.

Power system stability problem has received a great deal of attention over the last five decades. A large number of research papers and studies have been presented in the area of PSS in the literature [223]. Considerable efforts and researches have been directed towards designing optimal PSS that provides an optimal performance for a wide range of machine operating conditions and system parameters.

Tuning of PSS parameters is a difficult task because of the presence of poorly damped modes of oscillation and nonstop changes in operating conditions of a power system. Conventional PSS is commonly designed, with the phase compensation technique in the frequency domain and is implemented using lead-lag compensators whose parameters are determined based on a linearized model of the power system $[1-3]$.

The work presented in the three-part paper [2] developed the general concepts associated with applying PSSs utilizing shaft speed, ac bus frequency, and electrical power inputs and presented a discussion of the tuning concepts and practical aspects of stabilizer application and a general approach for analyzing PSSs. In [3], the researchers presented a detailed analytical effort to find out the parameters of PSSs for a large generating station, and reported small-signal and transient stability studies which confirmed the effectiveness of the stabilizers in enhancing the stability of inter-area and local plant models of oscillation. The researchers in [4] explored the phenomena of stability of synchronous machines under small perturbations by testing a SMIB system. In [5], the work provided an analysis of the performance of PSS under various system conditions and operating loads, and presented two approaches for the tuning of PSS parameters and investigated how PSS tuning and the dynamic performances of the system were affected by some factors. A fuzzy logic-based PSS with learning ability was proposed in [6], where the PSS employed a multilayer adaptive neural network, which was trained directly from the input and the output of the generating unit. The algorithm which combined the positive features of artificial neural networks and fuzzy logic control schemes provided good damping of power systems over a wide range of operating conditions and improved the dynamic performance of the system. The work presented in [7] introduced an approach for real-time tuning the parameters of a conventional PSS using a radial basis function network, which was trained using an orthogonal least squares learning algorithm. The work showed that the dynamic performance of the system using this approach was quite robust over a wide range of loading conditions and equivalent reactance. A robust design of multi-machine PSSs using simulated annealing (SA) optimization algorithm was presented in [8] to find optimal parameter settings of a conventional fixed-structure lead-lag PSS over a wide range of loading conditions and system configurations. The work presented an eigenvalue analysis and nonlinear simulation results and showed the effectiveness of the proposed design to damp out the local and the inter-area modes and considerably enhanced system stability. The research in [9] presented a method that simultaneously tuned multiple power system fixed lead-lag damping controllers using genetic algorithms, where modified GA operators were employed in the simultaneous optimization of both phase compensations and gain settings for the stabilizers. An optimal design with reduced computational burden of multi-machine PSSs using evolutionary programming was presented in [10] to determine optimal settings of PSS parameters that shifted the system eigenvalues of the electromechanical modes to the left side of the s-plane. The performance of the proposed approach under different disturbances, loading conditions, and system configurations was investigated and showed the effectiveness and robustness of the proposed designs to damp out the local and the inter-area modes of oscillations.

In [11] an approach was proposed that employed a PSO technique to determine robust optimal settings of PSS parameters in a multi-machine system, where two eigenvalue-based objective functions to enhance system damping of electromechanical modes were considered. Analysis and nonlinear simulation results presented in this work showed the effectiveness of the proposed scheme to damp out the local and inter-area modes of oscillations and work effectively over a wide range of loading conditions and system configurations. A robust tuning of classical lead-lag power systems stabilizers to enhance the stability of power systems operating at different loading conditions using eigenvalue 
analysis and simulation results was presented in [12], where the problem of selecting the stabilizer parameters was converted to a simple optimization problem with an eigenvalue-based objective function, which was solved by a tabu search algorithm. The researchers in [13] proposed a nonlinear variable structure stabilizer in a SMIB and a multi-machine system with multi-mode oscillations systems, where the design of this stabilizer involved a nonlinear transformation technique, a variable structure control technique and the linear system theory. Simulation results showed that the nonlinear variable structure stabilizer gave satisfactory dynamic performance and good robustness. The research in [14] described a controller, which could be realistically implemented using only local measurements for the generator excitation system that used a combination of feedback linearizing and the observation decoupled state space. The performance of the controller was consistent with respect to changes in network configuration, loading and power transfer conditions. A practical design for a stabilizer in multi-machine power system using the differential geometric linearization approach was presented in [15]. This stabilizer used the information at the secondary bus of the step-up transformer as input signals. The simulation results showed that the stabilizer granted good dynamic performance and robustness compared with conventional PSSs. An optimization technique to select the weighting matrix in linear optimal control system design, under the conditions of pre-specified closed-loop dominant eigenvalue locations and feedback gain limit constraints was presented in [16]. Simulation results of the stabilizer designed by the proposed technique indicated the effectiveness and correctness of the proposed technique.

An application of a multivariable self-tuning controller to a SMIB based on generalized predictive control under small and large disturbances was presented in [17]. The controller provided supplementary control signals to the excitation and the governor systems and showed the efficiency of the controller to damp low frequency oscillations in a power system. A simple and computationally efficient design of a self-tuning PSS using the pole-shifting technique was presented in [18]. The controller used a statefeedback law, whose gains were evaluated from the pole-shifting factor. The dynamic performance of the proposed PSS was adapted quickly to varying operating conditions. A robust flexible control strategy to design of PSS based on fuzzy logic and the sliding mode controller and a detailed sensitivity analysis were presented in [19]. The control objective was to enhance the stability and to improve the dynamic response of a SMIB system operating under wide variations in operating load and system parameters. The research in [20] presented a systematic approach and a detailed sensitivity analysis for the design of variable structure PSS, with desired eigenvalues in the sliding mode, which showed that the PSS was quite robust to wide variations in operating load and system parameters. A fuzzy logic PSS for a SMIB system was developed in [21] using speed and active power deviations as the controller input variables. This scheme did not require a plant model to design the PSS, but used neural network to tune PSS. The responses were compared with the fixed-parameter fuzzy PSS and a conventional (linear) power system stabilizer, showing that the neuro-fuzzy stabilizer was superior to both of them. A genetic algorithm approach to design an optimal fuzzy logic controller was introduced in [22]. The proposed method was used to design an optimal fuzzy logic excitation controller for a generating unit which showed very satisfactory results. An adaptive technique for the estimation of the synchronizing and damping torque coefficients of a synchronous machine using a linear adaptive neuron was introduced in [23] based on estimating the torque coefficients from online measurements of the changes of the rotor angle, rotor speed, and electromagnetic torque of the synchronous machine. The performance and advantages of the proposed technique over a wide range of operating conditions were compared with both Kalman filter and least-square error techniques.

Based on the idea that natural foraging strategy can lead to optimization, Passino proposed an evolutionary computation technique, which is the BF Optimization Algorithm [25, 26]. This idea is based on the concept that a foraging organism (or an animal) takes necessary action to maximize the energy intake per unit time spent for foraging, respecting all constraints imposed by its own physiology and environment where it exists. This BF stochastic search and optimization technique simulates the foraging behavior of $\mathrm{E}$. coli bacteria that commonly exists in intestines of human beings to locate, handle, and ingest food. The algorithm is an evolutionary algorithm as it integrates concepts of evolution and natural selection. In the last few years, BF optimization algorithm has been successfully applied to solve real world engineering optimization problems [26-34]. It has been shown in some study cases that BF performs better than other evolutionary computation algorithms such as genetic algorithm [38] and particle swarm optimization [39].

An application of BF for optimal location and parameters of a unified power flow controller by 
minimizing the objective function of the real power losses of a power system was presented in [28]. The problem was formulated as a non-linear constrained optimization problem. The results, which were compared with results obtained using interior point successive linearization technique, showed the superiority of the $\mathrm{BF}$ algorithm. In [29], the $\mathrm{FB}$ was used to optimize the coefficients of the proportional-integral controller for the active power filter and the obtained simulation results were compared with the GA. The comparisons showed quite satisfactory dynamic response and better convergence faster than GA to reach the global optimum solution. An application of BF Optimization for load frequency control of a power systems equipped with static synchronous series compensator was presented in [30]. The BF technique was used to optimize the optimal integral gain setting by minimizing quadratic performance index. Simulation results of the system including the compensator indicated that the deviations in area frequencies and inter area tie-line power were considerably improved in terms of peak deviations and setting time. A hybrid approach involving PSO and BF algorithm for optimal PSSs in a multi-machine power system was investigated in [31]. Simulation results showed the efficiency of the proposed hybrid technique to tune PSSs and demonstrated the effectiveness of the proposed controller to improve the power system stability over various conditions and disturbances. A hybrid approach involving GA and BF for tuning the PID controller of an automatic voltage regulator under different test cases was presented in [32]. Simulation results indicated that the suggested hybrid GA-BF system had better performance on obtaining the optimal parameters simultaneously. A dynamic BF algorithm to solve the optimal power flow problem with dynamic loads was presented in [33]. The proposed technique, which was examined and evaluated on the IEEE 30-bus test system and compared with the original version of BF algorithm and PSO algorithm, was able to adapt to various environmental changes. An approach for load forecasting using BF trained wavelet neural network was proposed in [34]. The proposed approach, which was validated by a real test system, was able to identify the inherent non-linear characteristics of power system loads.

In this paper the BF algorithm has been used to design an optimal PSS to improve stability, transient response and steady-state response of a SMIB system. The paper uses the integral of the time-weighted absolute error (ITAE) performance index for optimal controller design [24]. Moreover, the paper presents a comparison between the proposed BF-based optimal PSS design system, and GA-based and PSO-based optimal PSS design systems.

The remainder of the paper is organized as follows. Section 2 reviews the SMIB system modeling, PSS modeling, and basics of control systems related to the performance index used in the design process. In Section 3, a background on the classical BF optimization algorithm is presented. Section 4 provides simulation results of applying BF algorithm to the SMIB system and provides comparison between the results obtained and the results obtained by GA and PSO optimization algorithms. Finally conclusions are drawn in Section 5.

\section{Models of SMIB System and PSS}

\subsection{SMIB System}

A typical configuration of a SMIB system is shown in Fig. 1. The system represents a machine connected to a large system through a transformer and a transmission line. The Thevenin's equivalent of the transmission network external to the machine is used to obtain the representation of the system connected to the machine. Because of the relative size of the system to which the machine is supplying power, the dynamics associated with machine will cause virtually no change in the voltage and frequency of the Thevenin's voltage (infinite-bus voltage). In the figure, $X_{T}$ and $X_{L}$ represent the reactances of the transformer and the transmission line, respectively.

It is a common practice to use small signal analysis to study a power system response when a system is subjected to small perturbations. In small signal analysis, a linearized system model is used in control loop design. In this paper, the synchronous machine is described by the fourth-order model. The equations describing the steady-state operation of a synchronous generator connected to an infinite bus through an external reactance can be linearized about any particular operating point. The system dynamics can be expressed by a set of linear differential equations in the small-perturbation variables $\Delta \omega(t), \Delta \delta(t), \Delta E_{q}^{\prime}(t)$, and $\Delta E_{f d}(t)$ as follows [1-4]: 


$$
\begin{gathered}
\frac{d}{d t}(\Delta \omega(t))=-\frac{D}{2 H} \Delta \omega(t)-\frac{K_{1}}{2 H} \Delta \delta(t)-\frac{K_{2}}{2 H} \Delta E_{q}^{\prime}(t)+\frac{1}{2 H} \Delta T_{m}(t) \\
\frac{d}{d t}(\Delta \delta(t))=2 \pi f . \Delta \omega(t) \\
\frac{d}{d t}\left(E_{q}^{\prime}(t)\right)=-\frac{K_{4}}{T_{d o}^{\prime}} \Delta \delta(t)-\frac{1}{T_{d o}^{\prime} K_{3}} \Delta E_{q}^{\prime}(t)+\frac{1}{T_{d o}^{\prime}} \Delta E_{f d}(t) \\
\frac{d}{d t}\left(E_{f d}(t)\right)=-\frac{K_{A} K_{5}}{T_{A}} \Delta \delta(t)-\frac{K_{A} K_{6}}{T_{A}} \Delta E_{q}^{\prime}(t)-\frac{1}{T_{A}} \Delta E_{f d}(t)+\frac{K_{A}}{T_{A}} u(t)
\end{gathered}
$$

where,

$\omega$ Rotor angular speed of generator, p.u.

$\delta$ Rotor angle of generator, radians.

$D$ Mechanical damping torque coefficient of generator, p.u.

$E_{q}^{\prime}$ Transient voltage in the quadratic axis of the generator, p.u.

$E_{f d}$ Equivalent excitation (field) voltage, p.u.

$H$ Inertia constant, sec.

$T_{e}$ Electrical torque, p.u.

$T_{m}$ Mechanical torque, p.u.

$K_{A}$ Exciter gain.

$T_{A}$ Exciter time constant, sec.

$K_{1}$ Change in $T_{e}$ for a change in $\delta$ with constant flux linkages in the direct-axis

$K_{2}$ Change in $T_{e}$ for a change in direct-axis flux linkages with constant $\delta$.

$K_{3}$ Impedance factor

$K_{4}$ Demagnetizing effect of a change in rotor angle

$K_{5}$ Change in $V_{t}$ with a change in rotor angle for constant $E_{q}^{\prime}$

$K_{6}$ Change in $V_{t}$ with a change in $E_{q}^{\prime}$ constant rotor angle

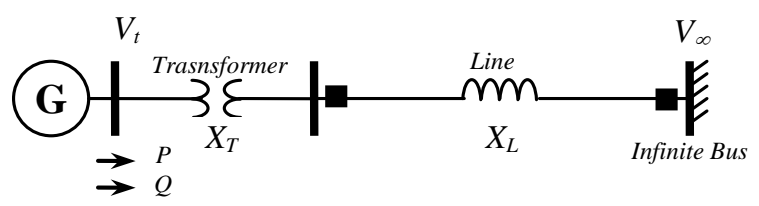

Figure 1. Schematic diagram of SMIB system

The interaction between the speed and voltage control equations of the machine is expressed in terms of six constants $K_{1}-K_{6}$. These constants, except $K_{3}$, are dependent on the actual real and reactive power loading as well as the excitation levels in the machine. $K_{3}$ is only a function of the ratio of impedance. Figure 2 shows the linearized Phillips-Heffron model of the single-machine connected to a large system (SMIB) around the operating point $[3,4]$.

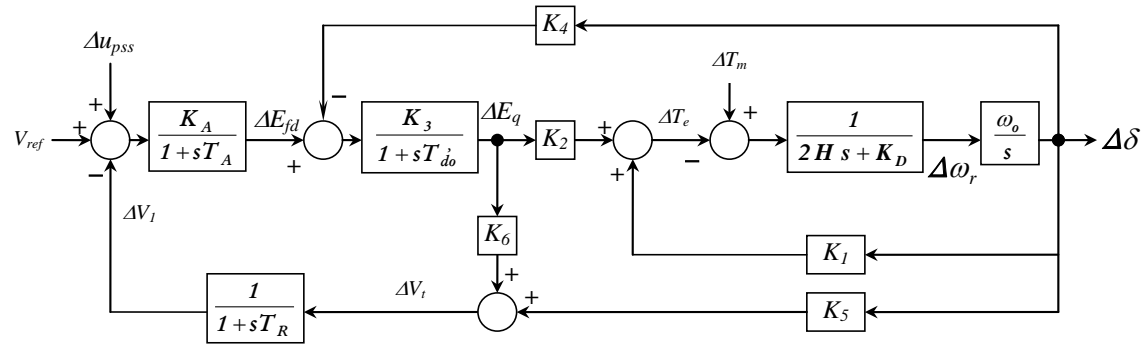

Figure 2. The block-diagram of SMIB Phillips-Heffron Model 
From the block diagram, the linearized differential equations of the SMIB system can be written in a state-space model as follows:

$$
\begin{gathered}
\frac{d}{d t} \Delta \boldsymbol{x}=A \Delta \boldsymbol{x}+B \Delta \boldsymbol{u} \\
\Delta \boldsymbol{y}=C \Delta \boldsymbol{x}+D \Delta \boldsymbol{u}
\end{gathered}
$$

The state variables are the deviation in rotor angle $(\Delta \delta)$, deviation in rotor speed $(\Delta \omega)$, deviation in voltage $\Delta E_{q}$ and deviation in field voltage $\Delta E_{f d}$. System state matrix $\mathrm{A}$ is a function of system parameters, which depends on operating conditions. Control matrix B depends on system parameters only. The vector of input (disturbance) signals to the system is $\Delta \boldsymbol{u}$. The input signals change in reference voltage $\left(\Delta V_{r e f}\right)$ and in mechanical torque $\left(\Delta T_{m}\right)$. The input $\Delta u_{p s s}$ shown in Fig. 2 is a control signal, which is the output of the PSS. In this paper the desired output signals under study are $\Delta \delta, \Delta \omega$, deviation in terminal voltage $\left(\Delta V_{t}\right)$ and deviation in electromagnetic torque $\left(\Delta T_{e}\right)$. Therefore, (5) and (6) can be expressed as:

$$
\begin{aligned}
\frac{d}{d t}\left[\begin{array}{c}
\Delta \delta \\
\Delta \omega \\
\Delta E_{q} \\
\Delta E_{f d}
\end{array}\right] & {\left[\begin{array}{cccc}
0 & \omega_{o} & 0 & 0 \\
\frac{-K_{1}}{2 H} & \frac{-D}{2 H} & \frac{K_{2}}{2 H} & 0 \\
\frac{-K_{4}}{T_{d o}^{\prime}} & 0 & \frac{-1}{T_{d o}^{\prime} K_{3}} & \frac{1}{T_{d o}^{\prime}} \\
\frac{-K_{A} K_{5}}{T_{A}} & 0 & \frac{-K_{A} K_{6}}{T_{A}} & \frac{-1}{T_{A}}
\end{array}\right]\left[\begin{array}{c}
\Delta \delta \\
\Delta \omega \\
\Delta E_{q} \\
\Delta E_{f d}
\end{array}\right]+\left[\begin{array}{cc}
0 & 0 \\
\frac{1}{2 H} & 0 \\
0 & 0 \\
0 & \frac{K_{A}}{T_{A}}
\end{array}\right]\left[\begin{array}{c}
\Delta T_{m} \\
\Delta V_{r e f}
\end{array}\right] } \\
& {\left[\begin{array}{c}
\Delta \delta \\
\Delta \omega \\
\Delta V_{t} \\
\Delta T_{e}
\end{array}\right]=\left[\begin{array}{cccc}
1 & 0 & 0 & 0 \\
0 & 1 & 0 & 0 \\
K_{5} & 0 & K_{6} & 0 \\
K_{1} & 0 & K_{2} & 0
\end{array}\right]\left[\begin{array}{c}
\Delta \delta \\
\Delta \omega \\
\Delta E_{q} \\
\Delta E_{f d}
\end{array}\right]+\left[\begin{array}{ll}
0 & 0 \\
0 & 0 \\
0 & 0 \\
0 & 0
\end{array}\right]\left[\begin{array}{l}
\Delta T_{m} \\
\Delta V_{r e f}
\end{array}\right] }
\end{aligned}
$$

The $K$-constants in (1)-(4), (7) and (8) are calculated as [4, 23]:

$$
\begin{gathered}
K_{1}=\frac{\left(x_{q}-x_{d}^{\prime}\right) i_{q o} E_{o} \sin \left(\delta_{o}\right)}{x_{e}+x_{d}^{\prime}}+\frac{E_{q o} E_{o} \cos \left(\delta_{o}\right)}{x_{e}+x_{q}} \\
K_{2}=\frac{E_{o} \sin \left(\delta_{o}\right)}{x_{e}+x_{d}^{\prime}} \\
K_{3}=\frac{x_{e}+x_{d}^{\prime}}{x_{e}+x_{d}} \\
K_{4}=\frac{\left(x_{d}-x_{d}^{\prime}\right) E_{o} \sin \left(\delta_{o}\right)}{x_{e}+x_{d}^{\prime}} \\
K_{50} \frac{x_{q} E_{o} \cos \left(\delta_{o}\right)}{x_{t o}+x_{q}}-\frac{v_{q o}}{V_{t o}} \frac{E_{o} \sin \left(\delta_{o}\right)}{x_{e}+x_{d}^{\prime}} \\
K_{6}=\frac{v_{q o}}{V_{t o}} \frac{x_{e}}{x_{e}+x_{d}^{\prime}}
\end{gathered}
$$

where,

$$
\begin{gathered}
i_{q o}=\frac{P_{o} V_{t o}}{\sqrt{\left(P_{o} x_{q}\right)^{2}+\left(V_{t o}^{2}+Q_{o} x_{q}\right)^{2}}} \\
v_{d o}=i_{q o} x_{q}
\end{gathered}
$$




$$
\begin{gathered}
v_{q o}=\sqrt{V_{t o}^{2}-V_{d o}^{2}} \\
i_{d o}=\frac{Q_{o}+x_{q} i_{q o}^{2}}{v_{q o}} \\
E_{q o}=v_{q o+} i_{d o} x_{q} \\
E_{o}=\sqrt{\left(v_{d o}+x_{e} i_{q o}\right)^{2}+\left(v_{q o}-x_{e} i_{d o}\right)^{2}} \\
\delta_{o}=\tan ^{-1}\left(\frac{v_{d o}+x_{e} i_{q o}}{v_{q o}-x_{e} i_{d o}}\right)
\end{gathered}
$$

and,

$i_{d}, i_{q} \mathrm{~d}$-axis and q-axis components of generator armature current, respectively, p.u.

$x_{d}, x_{d}^{\prime} \mathrm{d}$-axis synchronous and transient reactance, respectively, p.u.

$x_{q}, x_{q}^{\prime} \mathrm{q}$-axis synchronous and transient reactances, respectively, p.u.

$x_{e}$ Equivalent reactance of the system external to the machine terminals, p.u.

$v_{d}, v_{q} \mathrm{~d}$-axis and q-axis components of the generator terminal voltage, p.u.

$V_{t}$ Terminal voltage of the generator, p.u.

\section{$2.2 \quad$ PSS}

The PSS principally adds damping to the generator rotor oscillations by controlling its excitation using one or more of auxiliary stabilizing signals. To provide this damping function, the stabilizer generates a component of electrical torque in phase with the rotor speed deviations. PSS works on the idea of phase compensation by adjusting the PSS parameters to offset for phase lags through the generator, excitation system, and power system, such that PSS provides torque changes in phase with speed changes.

A conventional lead-lag PSS consists of three blocks: a phase compensation block, a signal washout block and a gain block [2-4]. The phase compensation block provides the suitable phase-lead characteristic to compensate for the phase lag between the exciter input and the generator electrical torque [2]. The washout block functions as a high-pass filter, which allows the dc signals to pass unchanged, thus avoiding terminal voltage variation due to steady changes in speed. The washout time constant $T_{W}$ is selected to be long enough to pass stabilizing signals at the frequencies of interest unchanged, but should not be so long that it causes undesirable generator voltage excursions during system-island conditions [3]. As the function of a PSS is to introduce a damping torque component, the speed deviation $(\Delta \omega)$ represents a suitable input signal for the PSS. In order to restrict the level of generator terminal voltage fluctuation during transient conditions, limits are imposed on PSS outputs. The block diagram of the lead-lag PSS is shown in Fig. 3, and the corresponding transfer function is given by:

$$
\begin{gathered}
G_{P S S}(s)=\frac{\Delta u_{p s s}}{\Delta \omega(s)}=K_{s t a b} \quad \frac{s T_{w}}{1+s T_{w}} \frac{1+s T_{1}}{1+s T_{2}} \frac{1+s T_{3}}{1+s T_{4}} \\
\Delta \omega(s) \rightarrow K_{s t a b} \rightarrow \frac{s T_{W}}{1+s T_{W}} \rightarrow \frac{1+s T_{1}}{1+s T_{2}} \rightarrow \frac{1+s T_{3}}{1+s T_{4}} \longrightarrow \Delta u_{p s s}(s)
\end{gathered}
$$

Figure 3. Transfer function block diagram for lead-lag PSS

In the PSS, $K_{s a t b}, T_{W}$, and $T_{1}-T_{4}$ are PSS stabilizing gain, washout time constant, and phase leadlag time constants, respectively. The parameters of the lead-lag PSS are required to be tuned optimally, in order to obtain the best performance of the system. Figure 4 shows the block diagram representation 
of the SMIB system equipped with a PSS.

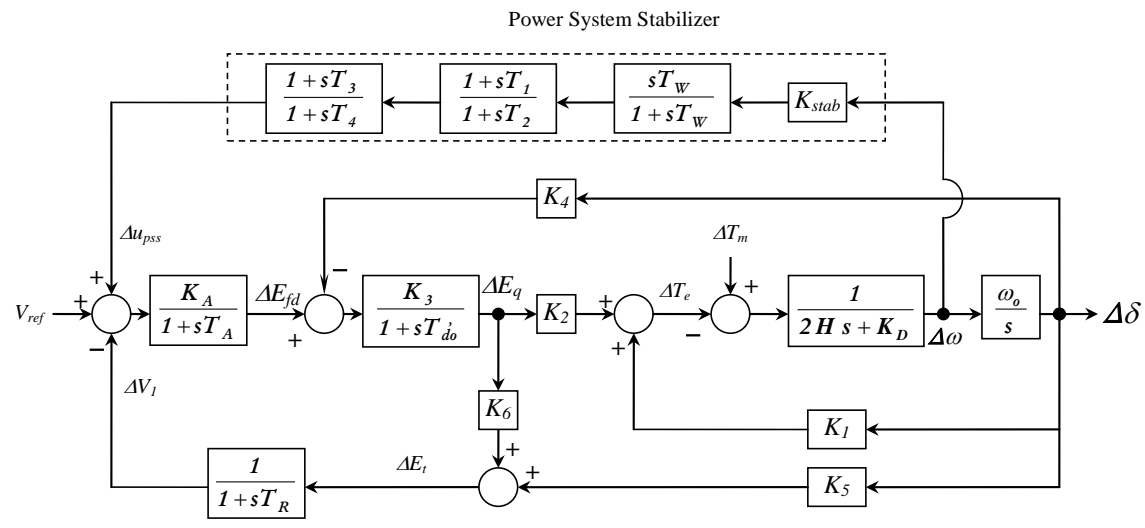

Figure 4. The block-diagram of SMIB Phillips-Heffron Model with PSS

The stabilizing gain $\left(K_{\text {stab }}\right)$ has an important effect on damping of rotor oscillations and indicates the amount of damping caused by the PSS, which ideally should be set to a value corresponding to maximum damping. Practically, $K_{\text {stab }}$ is set to a value that results in satisfactory damping of the critical system modes without compromising the stability of other modes, or transient stability, and that does not cause excessive amplification of PSS input signal noise [1-3].

The PSS parameters to be optimally tuned are the time constants $\left(T_{1}-T_{4}\right)$ and $K_{\text {stab }}$. Washout time constant $\left(T_{W}\right)$ should be long enough to pass stabilizing signals at the frequencies of interest relatively unchanged, but not so long that it leads to undesirable generator voltage excursions as a result of stabilizer action during system-islanding conditions. $T_{W}$ has a value in the range of 1 to 20 seconds. A washout time constant $T_{W}=10$ is used in the paper to ensure that the phase-lead and gain contributed by the washout block for the range of oscillation frequencies normally encountered is negligible.

In control system optimal design and analysis, performance indices are commonly used as quantitative measures to evaluate a system's performance, where a control system is judged as an optimal system when the system parameters are adjusted so that the index used in the design reaches its minimum value, while system constraints are respected. The commonly used indices are integral of the square of the error (ISE), integral of the absolute value of the error (IAE), integral of the time-weighted absolute error (ITAE), integral of time multiplied by the squared error (ITSE). If $e(t)$ indicates an error signal in the system, the ITAE measure, which will be implemented in this paper, is given by the following equation [24]:

$$
\text { ITAE }=\int_{0}^{\infty} t|e(t)| d t
$$

The optimal values for controller gains are obtained as the outputs of the constrained optimization of the ITAE performance measure (the objective function). The constraints are the lower and upper limits of the controller (PSS) parameters. The optimization problem can be formulated as:

$$
\text { Minimize ITAE }\left(K_{s t a b}, T_{1}, T_{2}, T_{3}, T_{4}\right)
$$

Subject to

$$
\begin{aligned}
& K_{\text {stab }}^{\min } \leq K_{\text {stab }} \leq K_{\text {stab }}^{\max } \\
& T_{1}^{\text {min }} \leq T_{1} \leq T_{1}^{\max } \\
& T_{2}^{\text {min }} \leq T_{2} \leq T_{2}^{\text {max }} \\
& T_{3}^{\text {min }} \leq T_{3} \leq T_{3}^{\max } \\
& T_{4}^{\text {min }} \leq T_{4} \leq T_{4}^{\max }
\end{aligned}
$$


where the objective function, ITAE in (23) and (24), is given by:

$$
\operatorname{ITAE}=\int t|\Delta \omega(t)| d t
$$

\section{Bacterial Foraging Optimization Algorithm}

The Bacterial Foraging (BF) algorithm, which is an evolutionary computational method, is based on the foraging behavior of the E. coli bacteria in human intestine. Foraging is a biological method in which an animal or bacterium moves in search of food and away from noxious elements. A basic part of the BF is the movement of the bacterium, where the motion is called chemotaxis, which is a motion that initiated by chemical stimulants. To enable motion, each bacterium has flagella (six rigid whip-like structures) that rotate to guide the types of motion exhibited by the bacterium in response to the stimuli it encounters within different mediums. Whilst searching for better forage, the E. coli alternately exhibits two distinct types of the chemotactic motion, which are tumble and swim (run) [25, 26].

In the $\mathrm{BF}$ algorithm, the motion of $\mathrm{E}$. coli is modeled according to the possible mediums the bacteria meet and their response within such mediums. Bacterium tumbles and runs alternately in the neutral substance medium. In a noxious substance medium, bacterium tumbles more than it swims as it tries to exit the noxious substance (to seek more favorable conditions). In nutrient substance medium, bacterium swims more than it tumbles while it searches for even more favorable nutrient mediums [25, $26]$.

The BF optimization algorithm is a social activity that requires collective search and foraging of a colony of bacteria, where it is necessary that each bacterium is able to communicate and harness this communication capability with other bacteria to fully utilize the collective searching and foraging of the colony. The communication for the bacteria colony of E. coli, is facilitated by the secretion of chemicals, which are termed attractants and repellents. Attractants are secreted to congregate bacteria to appropriate foraging spots, while repellents are secreted to alert other bacteria if inappropriate conditions have been encountered, thus alerting other bacteria to move away. Communication within the bacteria colony is modeled within the BF algorithm by implementing the swarming function. The $\mathrm{BF}$ optimization algorithm is summarized by the following stages: initialization, chemotaxis, swarming, reproduction and elimination/dispersal [25, 26].

\subsection{Initialization}

This stage includes three steps, which are [24, 25]:

- Initializing all parameters that are required for BF algorithm. These parameters are number of bacteria within the population $(S)$, positions of each bacterium within the sample space, number of chemotactic steps taken during each bacterium lifetime before reproduction $\left(N_{C}\right)$, number of reproduction event $\left(N_{r e}\right)$ and number of elimination/dispersal events $\left(N_{e d}\right)$ that would occur during the algorithm's implementation.

- The bacterial population is randomly distributed to different positions within the search space. Each bacterial position represents a potential solution to the optimization problem. The nutrient concentration function determines the nutrient concentration value for each bacterial position. The main target of the $\mathrm{BF}$ algorithm is to find the best solution that optimizes the nutrient concentration function. After the initialization phase, each bacterium searches for the best solution within the sample space by performing chemotaxis.

- For each initial bacterium position, use the nutrient concentration function to evaluate to a corresponding initial nutrient concentration value.

\subsection{Chemotaxis}

The E. coli bacterium has the tendency to gather at nutrient-rich areas by the chemotaxis activity, where bacteria move in response to chemical stimulus. While searching for the positions with best nutrient concentrations, each bacterium undergoes a specified number of chemotactic steps. The 
chemotaxis is achieved in two different modes: each bacterium can either move in a specified direction (run or swim), or it can move in a random direction (tumble). The bacteria always alternate between these two ways of chemotaxis all its life while searching for nutrients. In the chemotactic process, the tumble must always occur before the run $[25,26]$.

The BF optimization algorithm uses a nutrient concentration function for the search process. This function stands as an indicator of direction, which indicates improvement in the search procedure where this function quantifies nutrient concentration value at each bacterium position. The algorithm uses the initial nutrient concentration value as a reference to compare the subsequent nutrient concentration values that will be obtained during the algorithm's execution to decide which direction is good in the search. The concentration values are calculated each time a bacterium tumbles or runs from its previous position $[25,26]$.

Following a tumble, the new nutrient concentration value is determined and then compared with the initial nutrient concentration value. If the new value is better than the initial value, a run will follow in the direction randomly chosen by the tumble. On the other hand, if the new is worse than the initial value, there will be no run. The chemotactic process for this bacterium ends by storing the position and its corresponding nutrient concentration achieved. If the objective is to minimize the nutrient concentration function, then "better" means "smaller" and "worse" means "larger". At the end of each chemotactic step, the nutrient concentration is calculated. After a tumble or a run, the bacterial position $\theta^{i}(j, k, \ell)$ is given by $[25,26]$ :

where,

$$
\theta^{i}(j+1, k, \ell)=\theta^{i}(j, k, \ell)+C(i) \varphi(i)
$$

$$
\varphi(i)=\Delta(i) / \sqrt{\Delta^{T}(i) \Delta(i)}
$$

and

$i \quad$ A counter for each bacterium within the population,

$j$ A number of chemotactic steps that each bacterium has undertaken during its lifetime,

$k$ A counter for the reproduction steps,

$l$ An indicator for the elimination and dispersal event that is occurring,

$C(i) \quad$ A size of the chemotactic step taken in a random direction by the $i^{\text {th }}$ bacterium,

$\varphi(i) \quad$ A unit length random direction taken at each step, and

$\Delta(i) \quad$ A random vector $\in R^{P}$ with each element is a random number in the range $[-1,1]$.

\subsection{Swarming}

To enhance the effectiveness of the search and foraging procedure, when the E. coli bacteria are distributed randomly in a medium that has varying concentrations of nutrients or noxious substances, each bacterium produces attractants or repellents. The attractant is secreted as signal to attract other bacteria to areas with good nutrient concentration. This will result in the gathering of bacteria to form groups around areas with high nutrient concentration. On the other hand, repellent is secreted as a signal to redirect the search and foraging process away from the areas with noxious substances, where bacteria are advised to spread out to other areas. This swarming behavior of bacteria during the foraging process is modeled within the $\mathrm{BF}$ algorithm by the following cell-to-cell attraction/repulsion value $[25,26]$ :

$$
\begin{aligned}
& J_{c c}(\theta, P(j, k, \ell))=\sum_{i=1}^{S} J_{c c}^{i}\left(\theta, \theta^{i}(j, k, \ell)\right) \\
& =\sum_{i=1}^{S}-d_{\text {att }} \exp \left(-w_{\text {att }} \sum_{m=1}^{P}\left(\theta_{m}-\theta_{m}^{i}\right)^{2}+\sum_{i=1}^{S}-d_{\text {rep }} \exp \left(-w_{\text {rep }} \sum_{m=1}^{P}\left(\theta_{m}-\theta_{m}^{i}\right)^{2}\right)\right.
\end{aligned}
$$

where

$S$ Total number of bacteria,

$p \quad$ Number of parameters to be optimized which are present in each bacterium,

$d_{\text {att }}$ Quantification of the depth of the attractant released by each bacterium,

$w_{\text {att }}$ Quantification of the width of the attractant released by each bacterium, 
$d_{\text {rep }}$ Quantification of the depth of the repellent secreted by each bacterium, and

$w_{\text {rep }}$ Quantification of the width of the repellent secreted by each bacterium.

The cell-to-cell attraction/repulsion value, $J_{c c}(\theta, P(j, k, \ell))$, is added to the nutrient concentration function, which is to be optimized.

\subsection{Reproduction}

After chemotaxis step, reproduction step takes place. When every cell in the bacteria population has moved the specified number of chemotactic steps, reproduction process occurs. The reproduction means the transmission of genetic material from a donor cell to a recipient cell. In the reproduction process, the least healthy bacteria, which have a lower accumulated value of the objective function in one chemotactic lifetime, die and each of the other healthier bacteria splits into two to maintain a constant population size in the BF algorithm, which then starts exploring the search place from the same location. This process is necessary to cause all the bacteria within the population to converge at the bacteria population with the best nutrient concentration value [25, 26].

\subsection{Elimination and Dispersal}

The environment of a population of bacteria might be exposed to gradual or sudden changes which in turn might result in death of some of the bacteria population or cause general irregularities within the climate of the bacteria environment. Changes include variations in concentration of nutrients in the area the bacteria population exist due to their foraging habits or as a result of environmental conditions appearing or diminished nutrient concentration. The sudden or gradual changes in BF environment are modeled within the BF optimization algorithm by implementing elimination and dispersal events. The impact of dispersal event offsets the impact of elimination event. The elimination and dispersal events occur after a specified number of reproductive steps enhance the global search for the optimal solution $[25,26]$.

The elimination process is to randomly kill off some of the poorly performing bacteria within the population. The effect of elimination process is a decrease in the total number of bacterial population. Elimination is required to provide a space for new members of the bacterium population that are existed in areas with higher nutrient concentration.

The dispersal process follows the elimination process, where new bacteria members randomly take place the eliminated ones. The new ones are probably located in different locations, which are possibly better locations than the eliminated members of the population. The new locations might be better because they may be located nearer to spaces with better nutrient concentration. The net result is enhancing the global search procedure of the BF algorithm. It is worthwhile to mention that the elimination and dispersal events may have a negative effect on the foraging of the bacteria. This may occur if these events destroy bacteria in certain positions, which might be near nutrient-rich areas and transcribing parts of the bacterial population to locations with nutrient-poor concentration [25, 26].

\subsection{Termination}

The termination of the BF algorithm is based on a combination of criteria such as reaching convergence to a fixed low nutrient concentration value (fitness function value), reaching the maximum computation time for the algorithm, reaching a total number of elimination and dispersal events, and reaching a certain number of iterations. If the $\mathrm{BF}$ has reached a minimum value, the $\mathrm{BF}$ gives the position of the bacterium that corresponds to the optimal design of the controller [25, 26].

The details of the BF Optimization algorithm are presented in Algorithm 1. 
Algorithm 1. The BF optimization algorithm

Initialize all parameters required for BF algorithm: $S, p, N_{s}, N_{c}, N_{r e}, N_{e d}$, $P_{\text {ed }}, P(p, S, \ell), d_{\text {att }}, w_{\text {att }}, d_{\text {rep }}, w_{\text {rep }}, j=k=l=0$

Randomly distribute bacterial population to different positions within the search space

Use the nutrient concentration function to evaluate to a corresponding initial nutrient concentration value

for Elimination-dispersal loop do: $\ell=\ell+1$

for Reproduction loop do: $k=k+1$

for Chemotaxis loop do: $j=j+1$

for Bacterium $i=1$ : $S$

compute $J(i, j, k, l)$

Let $J_{s w}(i, j, k, l)=J(i, j, k, l)+J_{C C}\left(\theta^{i}(j, k, l), P(j, k, l)\right)$

Let $J_{\text {last }}=J_{\text {sw }}(i, j, k, l)$

end

for Bacterium $i=1: S$

Tumble:

Generate a random vector $\phi(i)$ as a unit length random direction

Move:

Let $\theta^{i}(j+1, k, l)=\theta^{i}(j, k, l)+C(i) \phi(i)$

Compute corresponding $J(i, j+1, k)$

Let $J_{s w}(i, j+1, k, l)=J(i, j+1, k, l)+J_{C C}\left(\theta^{i}(j+1, k, l), P(j+1, k, l)\right)$

Swim:

Let $m=0$

while $m<N_{\mathrm{s}}$ do

Let $m=m+1$

if $J_{s w}(i, j+1, k, l)<J_{\text {last }}$ then

$J_{\text {last }}=J_{\text {sw }}(i, j+1, k, l)$

$\theta^{i}(j+1, k, l)=\theta^{i}(j, k, l)+C(i) \phi(i)$

Compute corresponding $J(i, j+1, k, l)$

else

Let $m=N_{s}$

end

for the given $k$ and $l$, and for each bacterium $i=1,2, \ldots, S$ :

Calculate health of bacterium $i: J_{\text {health }}^{i}=\min _{j \in\left\{1,2, . ., N_{C}\right\}}\left\{J_{s w}(i, j, k, l)\right\}$

Sort bacteria in order of ascending cost $J_{\text {health }}$.

The $S_{r}=S / 2$ bacteria with the highest $J_{\text {health }}$ values die and other $S_{r}$ bacteria with the best values split.

end

for $i=1,2, \ldots, S$, eliminate and disperse the bacteria to random locations

on the optimization domain with probability $P_{e d}$

end 


\section{Simulation Results}

This section presents simulation results of the SMIB system using GA-based optimal PSS, PSO-based optimal PSS, and BF-based optimal PSS. A reader may refer to [35] for a background on GA and [36] for PSO. For each case of them, criterion of ITAE has been used to find the optimal values of PSS parameters. System data used in this paper are given in Table 1 [27]. Lower and upper limits of PSS parameters are given in Table 2 .

In order to investigate the effectiveness of the proposed BF-based optimal PSS, numerical simulations were carried out on the SMIB system shown in Fig. 3. In the simulations, two types of disturbances are used to test the system, which are:

(1) A step change in the reference terminal voltage $\left(\Delta V_{r e f}=0.1\right)$ at $t=0.1 \mathrm{sec}$, and

(2) A step change in the input mechanical power $\left(\Delta T_{m}=0.1\right)$ at $t=0.1 \mathrm{sec}$.

For each type of the above disturbances, the following three typical scenarios are considered: (1) No PSS (NC) is utilized, (2) BF-based optimal PSS (BFC), (3) GA-based optimal PSS (GAC), and (4) PSO-based optimal PSS (PSOC). By solving the appropriate optimization problems, the optimal stabilizer parameters for the system under study for the three test input (perturbation) signals are obtained for different input signals and summarized in Table 3.

As can be seen from the results presented in Table 3, the values of ITAE associated with the BFbased optimal PSS design are much smaller (better) than those obtained using the GA-based and PSObased optimal PSS designs. This fact will be reflected in the following response figures.

The response of the SMIB system, following a step change in load of 0.1 p.u. and a step change in voltage of 0.1 p.u. are shown in Figs. 5-12. For each of them, the responses represent deviation in speed $(\Delta \omega)$, deviation in angle $(\Delta \delta)$, deviation in terminal voltage $\left(\Delta V_{t}\right)$ and deviation in electrical toque $\left(\Delta T_{e}\right)$. Comparisons of NC, optimal BFC, optimal PSOC, optimal GAC obtained, following a step load change of 0.1 p.u, are shown in Figures 5-8. Comparisons of NC, optimal BFC, optimal PSOC, optimal GAC obtained, following a step change in voltage of 0.1 p.u, are shown in Figs. 9-12.

Table 1. System data of the SMIB system

\begin{tabular}{ll}
\hline Machine & $x_{d}=1.6, x_{d}^{\prime}=0.32, x_{q}=1.55, T_{d o}^{\prime}=6, D=0, H=5$ \\
Transmission line & $r_{e}=0, x_{e}=0.4$. \\
Exciter & $K_{A}=50, T_{A}=0.05$ \\
Operating point & $V_{t o}=1.0, P_{o}=1.0, Q_{o}=0.05, \delta_{o}=77.4^{o}, K_{1}=1.15839$, \\
& $K_{2}=1.43471, K_{4}=1.83643, K_{5}=-0.11133, K_{6}=0.31711$ \\
\hline
\end{tabular}

Table 2. Lower and upper limits of PSS parameters

\begin{tabular}{ccc}
\hline Parameter & Minimum Limit & Maximum Limit \\
\hline$K_{\text {stab }}$ & 0 & 100 \\
$T_{1}$ & 0.01 & 2.0 \\
$T_{2}$ & 0.01 & 2.0 \\
$T_{3}$ & 0.01 & 2.0 \\
$T_{4}$ & 0.01 & 2.0 \\
\hline
\end{tabular}


Table 3. Optimal PSS parameters for different perturbations

\begin{tabular}{|c|c|c|c|c|c|c|c|}
\hline Disturbance & Algorithm & $I T A E$ & $K_{s t a b}$ & $T_{1}$ & $T_{2}$ & $T_{3}$ & $T_{4}$ \\
\hline \multirow{3}{*}{ (1) Sudden change in $\Delta V_{r e f}$ only } & $\mathrm{BF}$ & 0.00133 & 20.15716 & 0.22119 & 1.71415 & 1.99215 & 0.05051 \\
\hline & GA & 0.00282 & 20.75602 & 0.14516 & 1.37265 & 1.45219 & 0.05290 \\
\hline & $\mathrm{PSO}$ & 0.00537 & 29.27035 & 0.68924 & 1.74262 & 1.28285 & 0.42248 \\
\hline \multirow{3}{*}{ (2) Sudden change in $\Delta T_{m}$ only } & $\mathrm{BF}$ & 0.00049 & 34.97769 & 0.24235 & 0.07281 & 1.49171 & 1.09790 \\
\hline & GA & 0.00056 & 41.15975 & 0.20563 & 1.93022 & 1.92186 & 0.06887 \\
\hline & $\mathrm{PSO}$ & 0.00277 & 24.26911 & 0.80417 & 0.15989 & 0.64777 & 1.93051 \\
\hline
\end{tabular}

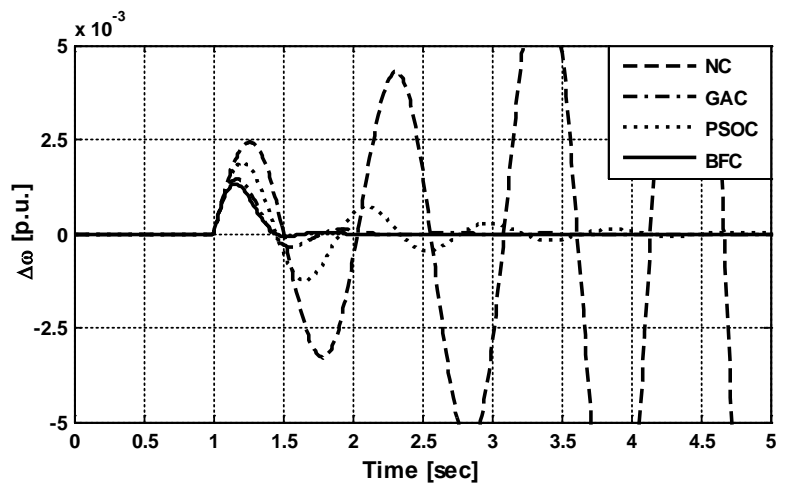

Figure 5. Comparison of $\Delta \omega$ for $\Delta T_{m}=0.1$ at $t=0.1 \mathrm{sec}$

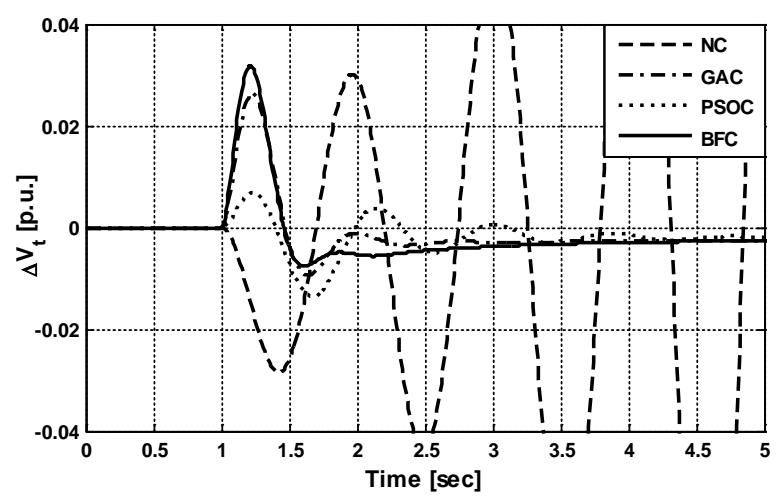

Figure 7. Comparison of $\Delta V_{t}$ for $\Delta T_{m}=0.1$ at $t=0.1 \mathrm{sec}$

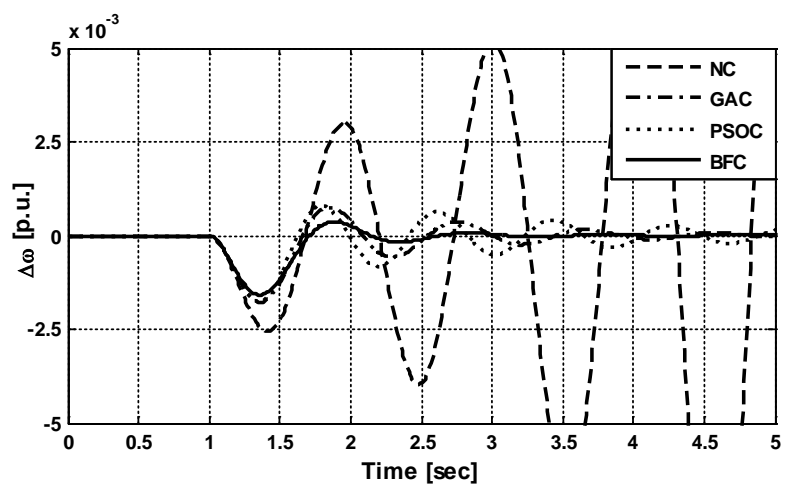

Figure 9. Comparison of $\Delta \omega$ for $\Delta V_{r e f}=0.1$ at $t=0.1 \mathrm{sec}$

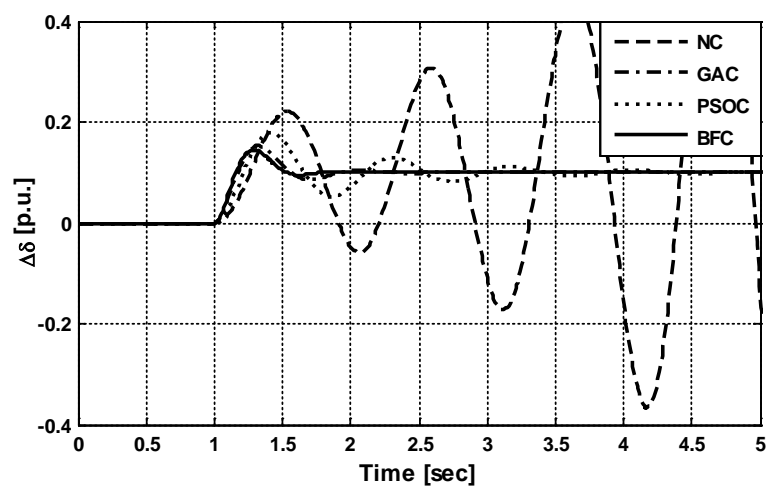

Figure 6. Comparison of $\Delta \delta$ for $\Delta T_{m}=0.1$ at $t=0.1 \mathrm{sec}$

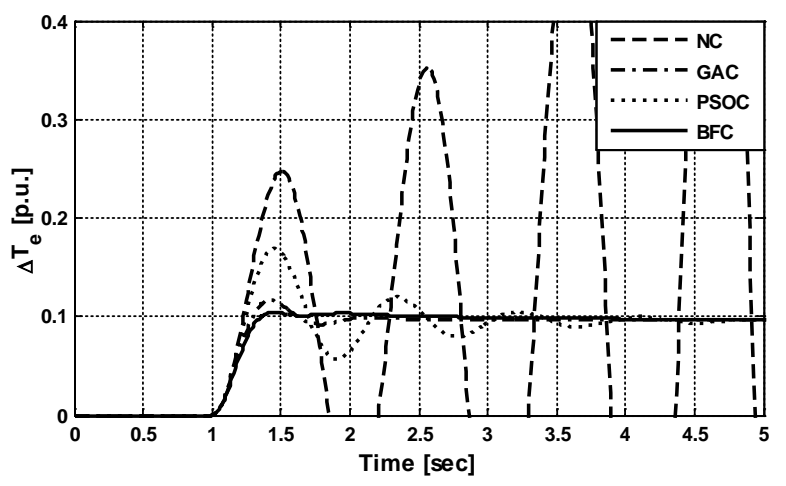

Figure 8. Comparison of $\Delta T_{e}$ for $\Delta T_{m}=0.1$ at $t=0.1 \mathrm{sec}$

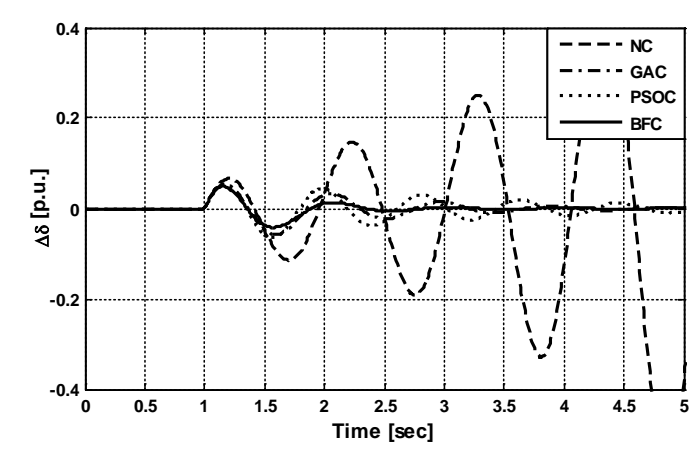

Figure 10. Comparison of $\Delta \delta$ for $\Delta V_{r e f}=0.1$ at $t=0.1 \mathrm{sec}$ 


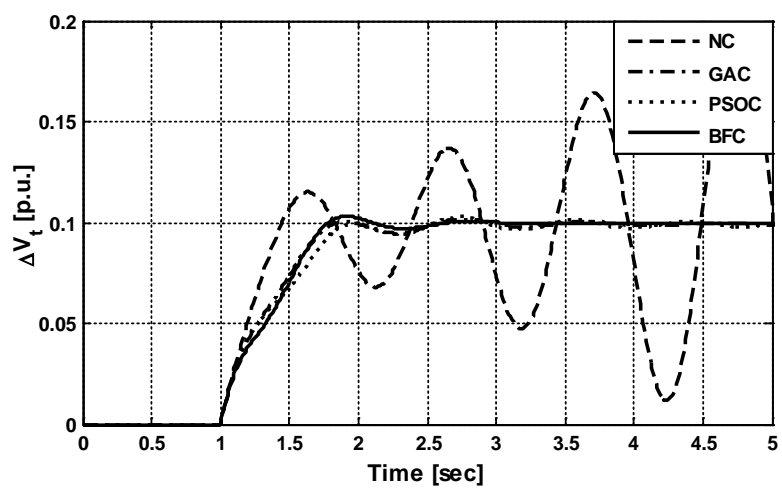

Figure 11. Comparison of $\Delta V_{t}$ for $\Delta V_{\text {ref }}=0.1$ at $t=0.1 \mathrm{sec}$

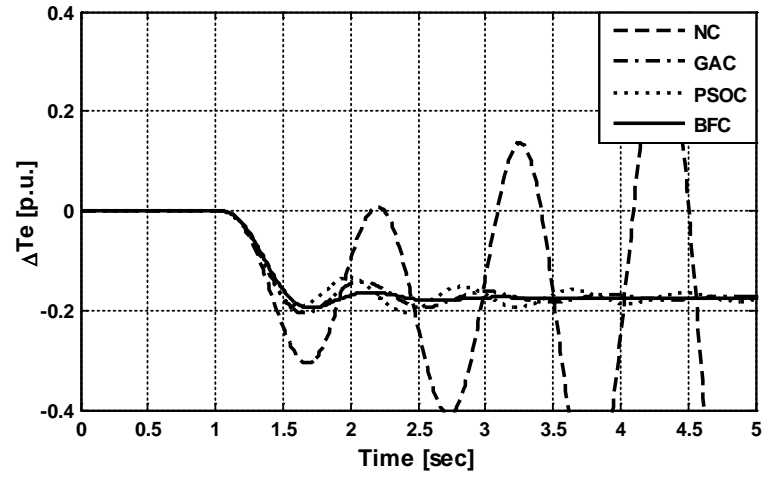

Figure 12. Comparison of $\Delta T_{e}$ for $\Delta V_{r e f}=0.1$ at $t=0.1 \mathrm{sec}$

\section{Discussion}

As can be seen from the results presented in Table 3, the minimum values of the objective function (ITAE), which indicates system's performance, determined by the BF-based optimal PSS design are much smaller than those determined by the GA-based and PSO-based optimal PSS designs. This fact indicates that BF gives better near-global solution than GA and PSO.

As the responses in the previous figures depict, the SMIB system, without a PSS controller, witnesses unstable operation for small perturbations as the responses obtained are growing-up functions. Evidently, the system would require a stabilizing signal from the PSS. If the SMIB system was stable without a PSS under smaller perturbations, the system would witness unsatisfactory long-persisting oscillations with considerable settling time, large overshoots and large steady-state errors.

The SMIB system with an optimally designed PSS becomes stable, but the system equipped with the optimal BF-based controller exhibits more satisfactory stabilized oscillations with much reduced settling time and overshoots compared to the GA-based and PSO-based designs of PSS. This fact is very clear from the results presented in Figures 5-12, which also reveal that optimal BF-based PSS controllers give much better results compared to those of the GA-based and PSO-based optimal controllers. This was also clear from the optimal values of the objective function given in Table 3. It can be noticed that with the optimal GA-based and PSO-based controllers, some disturbances still reveal undesired poorly stabilized performance of SMIB system

The responses shown in Figures 5-12, also demonstrate that the proposed BF-Based optimal PSS is more effective than the commonly used GA-based and PSO-based optimal PSS in damping the electromechanical oscillations of the rotor angle, rotational speed, electromagnetic torque, and terminal voltage under various disturbance conditions and its damping speed is considerably faster. All simulation results indicate that the BF-based optimal PSS is robust for all possible types of disturbances and achieves better performance in damping oscillations.

It can also be noted from Figures 5-12 that using the optimal BF-based optimal PSS can effectively improve both transient and steady-state behavior of the response compared to the cases of both GA and PSO-based optimal PSS designs. The significant improvement associated with the BF-based PSS is due to the fact that the algorithm of the BF leads to a better global solution of the optimization problem under study.

\section{Conclusions}

The PSS is a well-known and a widely used controller in power control systems as it is simple to realize and easy to tune. The PSS is utilized to dampen oscillations, increase stability and reduce steady-state error. This paper has proposed the design of power system stabilizer of SMIB system based on BF algorithm, which is an optimization algorithm that imitates the foraging behavior of E. coli bacteria. 
The objective is to enhance the stability of the system and to improve the dynamic and steady-state responses of the system operating in different conditions. Simulation results show that the optimal PSS obtained using BF optimization algorithm is better than the ones obtained using the two commonly used global optimization methods, GA and PSO, as the BF algorithm can give a better global or nearglobal solution of the optimal controller optimization problem, which is usually difficult to be obtained using the traditional optimization algorithms. The advantageous effects of applying BF algorithm to find the optimal parameters of the PSS have been explored. The results presented in the paper have shown that power system equipped with BF-based optimal controllers perform better than ones that have optimal controllers using some traditional optimization algorithms. Simulation results disclose that the optimal BF-based controller can significantly stabilize the system and can efficiently dampen oscillations under typical disturbances. The results presented in the paper indicate robustness and superiority of the proposed BF algorithm to solve the PSS design problem. Results also disclose that BF has the capability to attain a better solution with good quality convergence characteristics than some of the commonly global or near-global optimizers.

\section{References}

1. P. Kundur, Power system stability and control. McGraw-Hill, New York, 1994.

2. E. V. Larsen and D. A. Swann, "Applying power system stabilizers, Part I, II, III," IEEE Transactions on Power Apparatus and Systems, vol. 100, no. 6, pp. 3017-3046, 1981.

3. P. Kundur, M. Klein, G. J. Rogers, and M. S. Zywno, "Application of power system stabilizers for enhancement of overall system stability, "IEEE Transactions on Power Systems, vol. 4, no. 2, pp. 614-626, 1989.

4. F. De Mello and C. Concordia, "Concepts of synchronous machine stability as affected by excitation control," IEEE Transactions on Power Apparatus and Systems, vol. 88, no. 4, pp. 316-329, 1969.

5. K. Bhattacharya, J. Nanda, and M. L. Kothari, "Optimization and performance analysis of conventional power system stabilizers," International Journal of Electrical Power \& Energy Systems, vol. 19, no. 7, pp. 449-458, 1997.

6. A. Hariri and O. P. Malik, "A fuzzy logic based power system stabilizer with learning ability," IEEE Transactions on Energy Conversion, vol.11, no.4, pp.721-727, 1996.

7. R. Segal, M. L. Kothari, and S. Madnani, "Radial basis function (RBF) network adaptive power system stabilizer," IEEE Transactions on Power Systems, vol. 15, no.2, pp. 722-727, 2000.

8. M.A. Abido, "Robust design of multi-machine power system stabilizers using simulated annealing," IEEE Transactions on Energy Conversion, vol. 15, no.3, pp. 297-304, 2000.

9. A. L. B. Do Bomfim, G. N. Taranto, and D.M. Falcao, "Simultaneous tuning of power system damping controllers using genetic algorithms," IEEE Transactions on Power Systems, vol. 15, no.1, pp. 163-169, 2000.

10. M. A. Abido and Y. L. Abdel-Magid, "Optimal design of power system stabilizers using evolutionary programming," IEEE Transactions on Energy Conversion, vol. 17, no.4, pp. 429 - 436, 2002.

11. M. A. Abido, "Optimal design of power-system stabilizers using particle swarm optimization," IEEE Transactions on Energy Conversion, vol. 17, no.3, pp. 406 - 413, 2002.

12. Y. L. Abdel-Magid, M. A. Abido, and A. H. Mantaway, "Robust tuning of power system stabilizers in multimachine power systems," IEEE Transactions on Power Systems, vol. 15, no.2, pp. 735-740, 2002.

13. Y. Cao, L. Jiang, S. Cheng, D. Chen, O. P. Malik and G. S. Hope, "A nonlinear variable structure stabilizer for power system stability," IEEE Transactions on Energy Conversion, vol. 9, no.3, pp. 489 - 495, 1994.

14. J. W. Chapman, M. D. Ilic, C. A. King, and L. Eng, H. Kaufman, "Stabilizing a multimachine power system via decentralized feedback linearizing excitation control," IEEE Transactions on Power Systems, vol. 8, no.3, pp. 830-839, 1993.

15. M. Nambu and Y. Ohsawa, "Development of an advanced power system stabilizer using a strict linearization approach," IEEE Transactions on Power Systems, vol. 11, no. 2, pp. 813-818, 1996.

16. G. P. Chen and O. P. Malik, "Optimization technique for the design of a linear optimal power system stabilizer," IEEE Transactions on Energy Conversion, vo.7, no.3, pp. 453 - 459, 1992.

17. B. Wu and O. P. Malik, "Multivariable adaptive control of synchronous machine," IEEE Transactions on Power Systems, vol. 21, no.4, pp.1772-1781, 2006. 
18. M. L. Kothari, K. Bhattacharya, and J. Nanda, "Adaptive power system stabilizer based on pole-shifting technique," IEE Proceedings - Generation, Transmission and Distribution, vol. 143, no.1, pp. 96-98, 1996.

19. F. Rashidi, M. Rashidi, and H. Amiri, "An adaptive fuzzy sliding mode control for power system stabilizer," in Industrial Electronics Society, 2003, IECON'03. The 29th Annual Conference of the IEEE, vol. 1, pp. 626-630, 2003.

20. M. L. Kothari, J. Nanda, and K. Bhattacharya, "Design of variable structure power system stabilizer with desired eigen-values in the sliding mode," IEE Proceedings C - Generation, Transmission and Distribution, vol. 140, no.4, pp. 263-268, 1993.

21. N. Hossenizadeh and A. A. Kalam, "rule based fuzzy power system stabilizer tuned by a neural network," IEEE Transactions on Energy Conversion, vol. 14, no.3, pp.773-779, 1999.

22. J. Wen, S. Cheng and O. P. Malik, "A synchronous generator fuzzy excitation controller optimally designed with a genetic algorithm," IEEE Transaction on Power Systems, vol. 13, no.3, pp.884-889, 1998.

23. E. A. Feilat, "Fast estimation of synchronizing and damping torque coefficients using an adaptive neural network," in Proc. 42nd International University Power Engineering Conference, 4-6 September 2007, Brighton, United Kingdom, pp. 1049-1053.

24. D. Xue, Y. Chen, and D. P. Atherton, Linear feedback control: Analysis and design with MATLAB. SIAM, Philadelphia, 2007.

25. K. M. Passino, "Biomimicry of bacterial foraging for distributed optimization and control," IEEE Control Systems Magazine, vol. 22, no.3, pp. 52-67, 2002.

26. Y. Liu and K. M. Passino, "Biomimicry of social foraging bacteria for distributed optimization: models, principles, and emergent behaviors," Journal of Optimization Theory And Applications, vol.115, no.3, pp. 603$628,2002$.

27. D. H. Kim, A. Abraham, and J. H. Cho, "A hybrid genetic algorithm and bacterial foraging approach for global optimization," Information Sciences, vol. 177, no.18, pp. 3918-3937, 2007.

28. M. Tripathy, S. Mishra, L. L. Lai, and Q.P. Zhang, "Transmission loss reduction based on FACTS and bacteria foraging algorithm," in Proceedings of the Parallel Problem Solving from Nature, Reykjavik, Iceland, pp. 222-231, 2006.

29. S. Mishra and C.N. Bhende, "Bacterial foraging technique-based optimized active power filter for load compensation," IEEE Transactions on Power Delivery, vol. 22, no.1, pp. 457 - 465, 2007.

30. B. Paramasivam and I. A. Chidambaram, "Bacterial foraging optimization based load frequency control of interconnected power systems with static synchronous series compensator," International Journal of Latest Trends in Computing, vol. 1, no.2, pp. 7-13, 2010.

31. S. M. Abd-Elazim and E. S. Ali, "A hybrid particle swarm optimization and bacterial foraging for optimal power system stabilizers design," International Journal of Electrical Power E Energy Systems, vol. 46, no. 1, pp. 334-341, 2013.

32. D. H. Kim and J. H. Cho, "A biologically inspired intelligent PID controller tuning for AVR systems," International Journal of Control Automation and Systems, vol. 4, no.5, pp. 624-636, 2006.

33. W. J. Tang, M. S. Li, S. He, Q. H. Wu and J. R. Saunders, "Optimal power flow with dynamic loads using bacterial foraging algorithm," in Proc. 2006 Int. Conference on Power System Technology, Chongqing, China: 1$5,2006$.

34. M. Ulagammai, P. Venkatesh, P. S. Kannan and N. P. Padhy, "Application of bacterial foraging technique trained artificial and wavelet neural networks in load forecasting," Neurocomputing, vol. 70, no.16-18, pp. 26592667, 2007.

35. A. Wright, Genetic algorithms for real parameters optimization, foundations of genetic algorithms, Rawlines JE (Ed.), Morgan Kaufmann, San Mateo, CA, 1991.

36. R. C. Eberhart and J. Kennedy, "A new optimizer using particle swarm theory," in Proceedings of the Sixth International Symposium on Micromachine and Human Science, Nagoya, Japan, pp. 39-43, 1995. 